\title{
MAIZE GROWTH, YIELD FORMATION AND WATER-NITROGEN USAGE IN RESPONSE TO VARIED IRRIGATION AND NITROGEN SUPPLY UNDER SEMI-ARID CLIMATE
}

\author{
Umair ASHRAF ${ }^{1,2}$, Mazhar Noor SALIM ${ }^{3}$, Alam SHER ${ }^{4}$,Sabeeh-ur-Rasool SABIR ${ }^{5}$, \\ Aqil KHAN ${ }^{4}$, Shenggang PAN ${ }^{1,2}$, Xiangru TANG ${ }^{1,2 *}$ \\ ${ }^{1}$ South China Agricultural University, College of Agriculture, Department of Crop Science and \\ Technology, Guangzhou, CHINA \\ ${ }^{2}$ Scientific Observing and Experimental Station of Crop Cultivation in South China, Ministry of \\ Agriculture, P.R. China, Guangzhou, CHINA \\ ${ }^{3}$ University of Agriculture, Department of Agronomy, Faisalabad, PAKISTAN \\ ${ }^{4}$ Anhui Agriculture University, College of Agronomy, Department of Crop Cultivation and Farming \\ System, Anhui, CHINA \\ ${ }^{5}$ Lanzhou University, School of Life Sciences, State Key Laboratory of Grassland Agroecosystem, \\ Lanzhou, CHINA \\ *Corresponding author: tangxr@scau.edu.cn
}

Received: 29.06.2015

\begin{abstract}
Enhancement of resource use efficiencies and crop productivity in sustainable agriculture are important especially in low-input production systems. This experiment was planned to evaluate the performance of maize under different water and nitrogen levels. Three irrigation treatments i.e., $I 1=$ three leaf stage $(V 1)$, nine leaf stage (V2), tasseling (T) and milking stage (M); I2 = V1, V2, T, M and dough stage (R1), I3= V1, V2, T, M , R1 and blister stage (R2) were applied with $70 \mathrm{~mm}$ application depth with different nitrogen application rates i.e., $0,150,200$ and $250 \mathrm{~kg} \mathrm{~N} \mathrm{ha}^{-1}$. Results revealed that maximum growth i.e., plant height, leaf area index (LAI), crop growth rate (CGR), cob length and diameter as well as yield and yield components i.e., grains rows/cob, grains/row, grains/cob, grain weight/cob, 100-grain weight, grain yield, biological yield and harvest index, water and nitrogen use efficiencies as well as transpiration and photosynthetic activities were recorded at $I_{3}$ with $250 \mathrm{~kg} \mathrm{~N} \mathrm{ha}^{-1}$. However, increased irrigation and nitrogen application rates delayed days to tasseling, silking and maturity. Conclusively, six irrigations $\left(I_{3}\right)$ with $250 \mathrm{~kg} \mathrm{~N} \mathrm{ha}^{-1}$ can be adopted as the best input levels to get maximum maize yield under semi-arid regions. In future both these inputs may be used as water and nitrogen based agricultural best management practices (BMPs) in regions with similar type of environmental conditions.
\end{abstract}

Keywords: Growth, Maize, Resource use efficiency, Photosynthesis, Respiration, Yield.

\section{INTRODUCTION}

Maize (Zea mays L.) is an important cereal crop grown all over the world under a diverse type of climate. Besides agronomic, edaphic and other management factors, prevailing climatic conditions of a specific region or local climate also affects its growth and overall productivity ( $\mathrm{Li}$ et al., 2010; Mohamed, 2010). Management of irrigation water and nitrogen is crucial in order to improve maize productivity with reduced pollution risks (Gheysari et al., 2009) as both of these factors had a positive correlation with maize productivity and can induce yield loss if applied in an inappropriate way (Di Paolo and Rinaldi, 2008). Effects of irrigation and nitrogen application on growth, yield, irrigation water use efficiency (IRRWUE), water use efficiency (WUE), nitrogen use efficiency
(NUE) has been well reported in previous literature (Khaliq et al., 2009; Sepaskhah et al., 2011; Hammad et al., 2012). They recorded highest maize yield with a thorough restitution of crop evapotranspiration even though WUE and IRRWUE decreases with increase in irrigation water. However, a linear increase in WUE with respect to crop actual/potential evapotranspiration was also observed by Payero et al. (2006) in the semi-arid conditions of the US Great Plains.

A significant reduction in maize yield due to water deficit even at high doses of nitrogen has been reported by Moser et al. (2006). Morpho-physiological and biochemical attributes of maize has been reduced when maize is subjected to drought conditions at tasseling stage (Anjum et al., 2011). Moreover, a strong relationship 
exists between soil moisture level and $\mathrm{N}$ uptake by Aynehband et al. (2011). N-deficit conditions hampered light interception by reducing leaf area, hence abridged photosynthetic activity and led to yield penalty (Glamoclija et al., 2011). Nonetheless, maize is somewhat tolerant to $\mathrm{N}$-deficit conditions at early vegetative stage than later reproductive phase (Islam et al., 2010) while Halvorson et al. (2006) described maize yield as a function of available water and nitrogen.

$\mathrm{N}$ application in proper dose at a right time is a single most significant component in agricultural systems to improve crop production (Khaliq et al., 2009). Hammad et al. (2011) concluded from his findings that nitrogen at 200 $\mathrm{kg} \mathrm{N} \mathrm{ha-1}$ for maize is not enough to get optimal yields under semi-arid conditions. In this regard, agricultural best management practices (BMPs) can effectively be used for resource management in successful crop production systems (Abbas and Fares, 2009). So, formulation of water and nitrogen based BMPs are required in this era to balance application and requirements of these two important factors to sustain crop productivity without damaging the environment. To establish water and $\mathrm{N}$ based BMPs, there is a need to determine optimal dosages of these two inputs for successful production of maize under semi-arid conditions. Till now a little work has been done in this regard specifically under semi-arid climatic conditions. This study was therefore initiated to evaluate the performance, yield response and water-nitrogen use efficiencies of maize under different levels of water and nitrogen.

\section{MATERIALS AND METHODS}

\section{Site Description and Treatments}

The experiment was conducted at Agronomic Research Farm, University of Agriculture, Faisalabad, Pakistan $\left(31^{\circ} 25^{\prime} \mathrm{N}, 73^{\circ} 04^{\prime} \mathrm{E}\right)$ during autumn seasons (July to October) of 2013 and 2014. The climate of this region is semi-arid and subtropical. Normally, the weather of this region ranges between $-1{ }^{\circ} \mathrm{C}$ (in January) and $48{ }^{\circ} \mathrm{C}$ (in June) with mean annual rainfall about 200-250 mm and falls in semi-arid region. Most of the rainfall in this region occurs during monsoon season (July-August). Further, prevailing climatic conditions during the crop growth season is presented in figure 1. The experiment comprised of three different irrigation schedules i.e., $\mathrm{I}_{1}=$ three leaf stage (V1), nine leaf stage (V2), tasseling (T) and milking stage $(\mathrm{M}) ; \mathrm{I}_{2}=\mathrm{V} 1, \mathrm{~V} 2, \mathrm{~T}, \mathrm{M}$ and dough stage $(\mathrm{R} 1), \mathrm{I}_{3}=$ $\mathrm{V} 1, \mathrm{~V} 2, \mathrm{~T}, \mathrm{M}, \mathrm{R} 1$ and blister stage (R2) and four nitrogen levels $\left(\mathrm{N}_{0}=0 \mathrm{~kg} \mathrm{~N} \mathrm{ha}^{-1}, \mathrm{~N}_{\mathrm{I}}=150 \mathrm{~kg} \mathrm{~N} \mathrm{ha}^{-1}, \mathrm{~N}_{2}=200 \mathrm{~kg}\right.$ $\mathrm{N} \mathrm{ha}^{-1}$ and $\mathrm{N}_{3}=250 \mathrm{~kg} \mathrm{~N} \mathrm{ha}^{-1}$ ). Irrigation treatments were applied with an application depth of $70 \mathrm{~mm}$.

a) $\square$ Rainfall (mm) $\quad$-Daily max. temp. ${ }^{\circ} \mathrm{C} \quad-$ - Daily min. temp. ${ }^{\circ} \mathrm{C}$

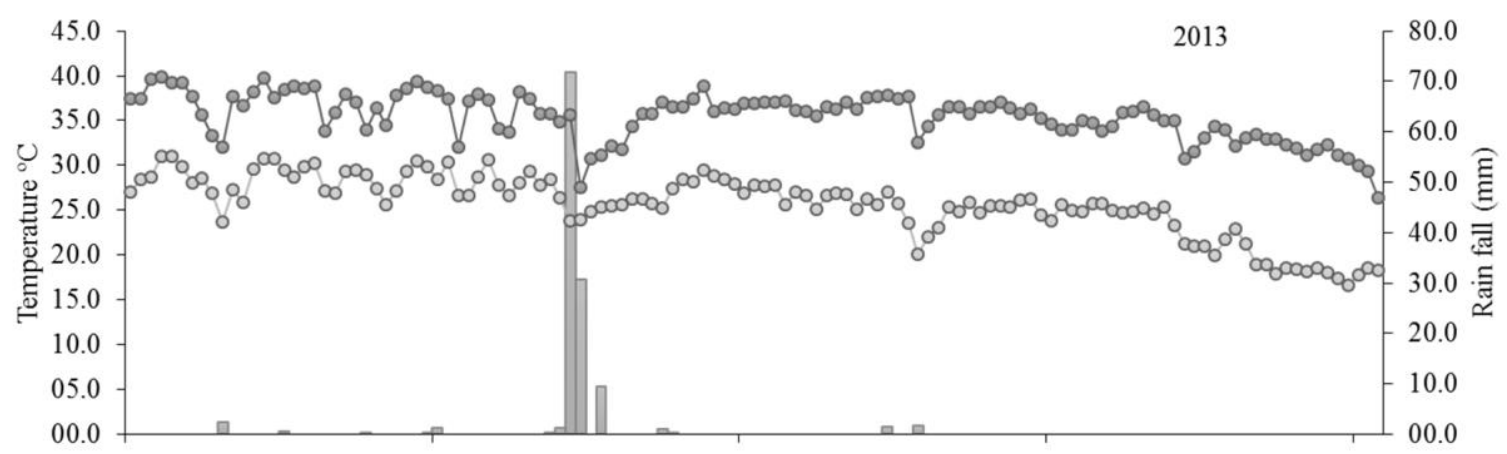

b)

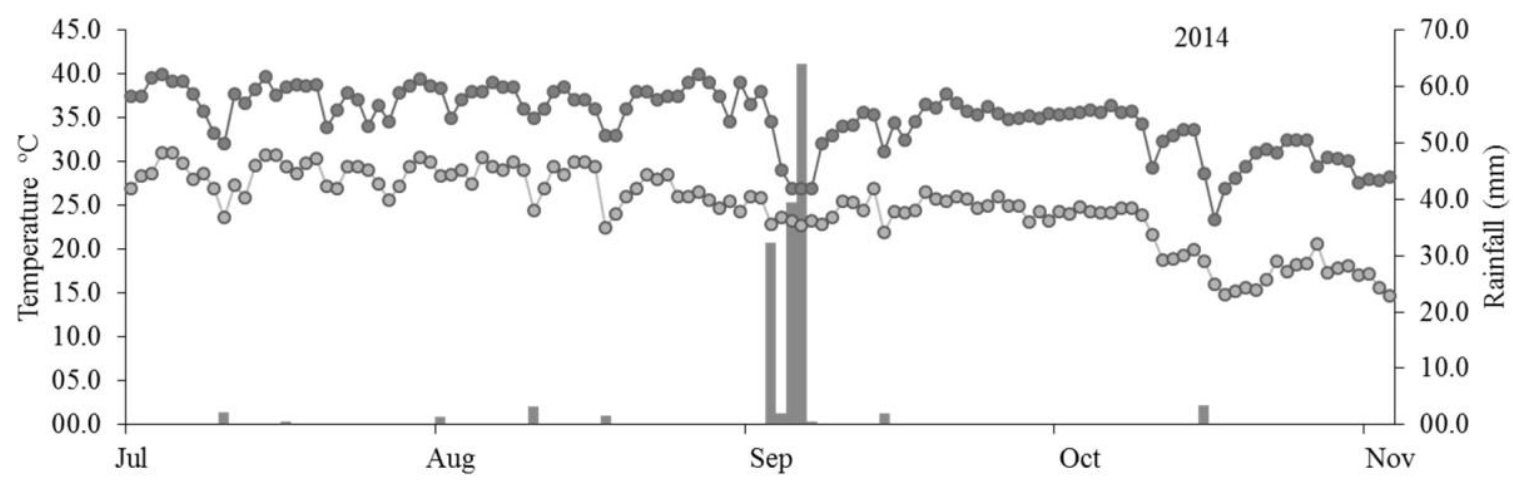

Figure 1. Prevailing climatic conditions of the experimental site during crop growing seasons for the year (a) 2013 and (b) 2014. 


\section{Soil Analysis}

To determine major physical and chemical properties of soil, composite soil samples were taken from above soil surface $(0-30 \mathrm{~cm})$ at the experiment site prior to sowing. Collected samples were chemically analyzed by following the protocols advised by Homer and Pratt (1961). The soil was loamy containing sand $(38 \%)$, silt $(46 \%)$ and clay (16\%) particles, having bulk density $\left(1.44 \mathrm{~g} \mathrm{~cm}^{-3}\right)$, field capacity $\left(0.35 \mathrm{~cm}^{3} \mathrm{~cm}^{-3}\right)$, saturation $(36 \%), \mathrm{pH}(7.50), \mathrm{EC}$ $\left(1.06 \mathrm{dSm}^{-1}\right)$, organic matter $(0.98 \%)$, available nitrogen $(0.055 \%)$, available phosphorus (10.8 ppm) and available potassium (180 ppm).

\section{Crop Husbandry}

In both growing seasons, hybrid maize " $R-2303$ ', was planted on $75 \mathrm{~cm}$ apart ridges during the second week of July by using the seed rate of $25 \mathrm{~kg} \mathrm{ha}^{-1}$. Plant $\times$ plant distance was maintained $20 \mathrm{~cm}$ by thinning out extra plants at four leaf stage to keep optimum plant population. Nitrogen, phosphorous and potash were applied in accordance with treatments in the form of urea, single super phosphate (SSP) and sulphate of potash (SOP) respectively. Phosphorus and potassium were applied at $135 \mathrm{~kg} \mathrm{ha}^{-1}$ and $115 \mathrm{~kg} \mathrm{ha}^{-1}$, respectively at sowing time. The nitrogen was applied in three split forms as half of the nitrogen was applied at sowing time while remaining dose was in two splits according to the treatments under study. Weeds were manually controlled by hoeing. Seed were treated with fungicide (Benlate at $2 \mathrm{~kg} \mathrm{ha}^{-1}$ ) for protection against seed-borne fungi. Canal irrigation water $(\mathrm{EC}=$ $0.56 \mathrm{mS} \mathrm{cm}^{-1}$, total soluble salts $(\mathrm{TSS}=375)$ was applied according to schedule by using surface irrigation system. Cut throat flume method was followed to measure the depth of applied water as described by Hammad et al. (2012) by using the following formula:

$$
\mathrm{t}=(\mathrm{A} \times \mathrm{d}) \div \mathrm{Q}
$$

Where, $\mathrm{t}=$ time to irrigation $(\mathrm{s}), \mathrm{Q}=$ discharge $\left(\mathrm{m}^{3} \mathrm{~s}\right.$ $\left.{ }^{1}\right), A=$ area $\left(\mathrm{m}^{2}\right)$ and $\mathrm{d}=$ depth of water $(\mathrm{mm})$. Water use efficiency (WUE) was calculated as:

Grain yield /Total water (evapotranspiration) consumed by the crop during whole season.

The crop was harvested manually in the end of October. After harvesting, the plants are tied into bundles and stalked for one week. After that the cobs were removed from the stalk and allowed to dry in sunlight for two weeks before shelling.

\section{Observations}

Five plants were selected from each subplot at random, tagged and continuously visited for recording the number of days to tasseling, silking and maturity taken by the plants. Leaf area was measured at tasseling with the help of leaf area meter (CI-202, CID Bio-Science); and the leaf area index (LAI) was calculated by using a standard formula described by (Watson, 1952). Likewise, crop growth rate (CGR) was calculated following the method described by Hunt (1978). At maturity, 10 plants were sampled from to determine various growth, yield and yield related attributes. Harvest index (HI) was calculated by the following the formula:

$$
\mathrm{HI}=\frac{\text { Grain yield }}{\text { Biological yield }} \times 100
$$

Nitrogen use efficiency (NUE) was calculated by the formula described Dobermann (2007).

$$
\mathrm{NUE}=\frac{\mathrm{Y}-\mathrm{YO}}{\mathrm{F}}
$$

Where $\mathrm{Y}$ and $\mathrm{Y} 0$ are the grain yields $\left(\mathrm{kg} \mathrm{ha}^{-1}\right)$ with applied $\mathrm{N}$ and without $\mathrm{N}$, respectively while $\mathrm{F}$ is the amount of $\mathrm{N}$ applied $\left(\mathrm{kg} \mathrm{ha}^{-1}\right)$.

\section{Experimental Design and Statistical Analyses}

The experiment was laid out in a randomized complete block design (RCBD) with split plot arrangement having three replications with a net plot size of $7 \mathrm{~m} \times 3 \mathrm{~m}$. Irrigation schedules were randomized in main plots and nitrogen levels in sub plots. Each treatment was repeated three times in both years. In the combined analysis of data, the interaction of year and treatments was nonsignificant; thus, the data were pooled for both years (2013-2014) and presented with the interaction of irrigation and nitrogen application rates. The recorded data were statistically analyzed by Statistix version 8 (Analytical, Tallahassee, Florida, USA) while least significant difference (LSD) test at $5 \%$ probability was used to compare treatment means. Graphs for experimental data were generated by using SigmaPlot 9.0 (Systat Software, Inc., San Jose California USA) while MS Excel 2013 was used to represent the climatic data. The relationships between variables were estimated by using polynomial linear regression analyses.

\section{RESULTS}

Maize phenology, growth, yield and yield related attributes were significantly $(\mathrm{p} \leq 0.05)$ affected by irrigation dynamics and nitrogen application rates. Plants took more days to tasseling, silking and maturity at $\mathrm{I}_{3}$ level of irrigation followed by $\mathrm{I}_{2}$ and $\mathrm{I}_{1}$ levels. Likewise increased application rates of nitrogen delayed the maturity of maize plants. Maximum number of days taken by plants for tasseling, silking and maturity were recorded at $\mathrm{N}_{3}$ level of nitrogen application while minimum days were recorded in control. The overall trend (days from max. to min.) of nitrogen application rates regarding maize phenological stages were recorded as: $\mathrm{N}_{3}>\mathrm{N}_{2}>\mathrm{N}_{1}$ $>\mathrm{N}_{0}$. However, the interaction of irrigation $\times$ nitrogen regarding maize phenology was insignificant (Table 1). 
Table 1. Effect of different irrigation and nitrogen levels on maize phenology

\begin{tabular}{|c|c|c|c|}
\hline Treatments & Days to tasseling & Days to silking & Days to maturity \\
\hline $\mathrm{I}_{1}$ & $45.93 \pm 1.67 \mathrm{a}$ & $54.20 \pm 1.67 \mathrm{~b}$ & $87.22 \pm 1.33 \mathrm{c}$ \\
\hline $\mathrm{I}_{2}$ & $46.87 \pm 0.99 \mathrm{ab}$ & $55.17 \pm 0.90 \mathrm{ab}$ & $94.85 \pm 1.58 \mathrm{~b}$ \\
\hline $\mathrm{I}_{3}$ & $49.33 \pm 1.01 \mathrm{a}$ & $55.83 \pm 1.11 \mathrm{a}$ & $100.85 \pm 2.07 \mathrm{a}$ \\
\hline $\mathrm{LSD}(\mathrm{p} \leq 0.05)$ & 1.14 & 1.10 & 0.49 \\
\hline $\mathrm{N}_{0}$ & $46.75 \pm 0.99 \mathrm{~d}$ & $53.80 \pm 0.98 \mathrm{~d}$ & $89.15 \pm 1.02 \mathrm{~d}$ \\
\hline $\mathrm{N}_{1}$ & $48.11 \pm 0.67 \mathrm{c}$ & $54.15 \pm 0.67 \mathrm{c}$ & $93.28 \pm 1.49 \mathrm{c}$ \\
\hline $\mathrm{N}_{2}$ & $49.31 \pm 1.00 \mathrm{~b}$ & $55.35 \pm 1.02 \mathrm{~b}$ & $95.72 \pm 1.24 \mathrm{~b}$ \\
\hline $\mathrm{N}_{3}$ & $50.55 \pm 1.01 \mathrm{a}$ & $56.60 \pm 1.10 \mathrm{a}$ & $99.18 \pm 1.20 \mathrm{a}$ \\
\hline $\mathrm{LSD}(\mathrm{p} \leq 0.05)$ & 0.50 & 0.48 & 0.82 \\
\hline Irrigations & $*$ & $*$ & $*$ \\
\hline nitrogen & $*$ & $*$ & $*$ \\
\hline Irrigations $\times$ nitrogen & NS & NS & NS \\
\hline
\end{tabular}

Individual effects of all irrigation and nitrogen application rates were significant for cob length, cob diameter, grain rows/cob, grains/row, grains/cob and grain weight/cob. The results revealed that $I_{3}$ level of irrigation were remained more effective than $\mathrm{I}_{2}$ and $\mathrm{I}_{1}$. $\mathrm{I}_{3}$ resulted in longer $(11.14 \%)$ and thick cobs $(14.5 \%)$, produced more number of grain rows/cob $(5.71 \%)$, grains/row $(9.38 \%)$ and grain weight per cob $(7.61 \%)$ compared with $I_{1}$. Further, significant improvements of these parameters were observed at higher nitrogen application rates. The affect was linearly increased with the doses of nitrogen applied. $\mathrm{N}_{3}$ remained the most effective for cob formation and grain development in maize followed by $\mathrm{N}_{2}, \mathrm{~N}_{1}$ and $\mathrm{N}_{0}$ (Table 2).

Table 2. Cob length, cob diameter, grains rows/cob, grains/row, grains/cob and grain weight/cob of maize as affected by different irrigation and nitrogen levels

\begin{tabular}{lcccccc}
\hline Treatments & $\begin{array}{c}\text { Cob length } \\
(\mathbf{c m})\end{array}$ & $\begin{array}{c}\text { Cob diameter } \\
(\mathbf{c m})\end{array}$ & $\begin{array}{c}\text { Grains } \\
\text { rows/cob }\end{array}$ & Grains/row & Grains/cob & $\begin{array}{c}\text { Grain } \\
\text { weight/cob }(\mathbf{g})\end{array}$ \\
\hline $\mathrm{I}_{1}$ & $14.30 \pm 0.36 \mathrm{c}$ & $2.49 \pm 0.21 \mathrm{c}$ & $14.12 \pm 0.63 \mathrm{~b}$ & $27.46 \pm 2.19 \mathrm{~b}$ & $399 \pm 6.62 \mathrm{~b}$ & $98.09 \pm 2.60 \mathrm{c}$ \\
$\mathrm{I}_{2}$ & $15.08 \pm 0.31 \mathrm{~b}$ & $2.61 \pm 0.13 \mathrm{~b}$ & $14.70 \pm 1.53 \mathrm{ab}$ & $29.01 \pm 2.65 \mathrm{a}$ & $441 \pm 8.93 \mathrm{a}$ & $113.5 \pm 5.97 \mathrm{~b}$ \\
$\mathrm{I}_{3}$ & $16.15 \pm 0.26 \mathrm{a}$ & $2.74 \pm 0.18 \mathrm{a}$ & $14.94 \pm 2.07 \mathrm{a}$ & $30.15 \pm 2.46 \mathrm{a}$ & $464 \pm 5.24 \mathrm{a}$ & $127.6 \pm 4.90 \mathrm{a}$ \\
\hline $\mathrm{LSD}(\mathrm{p} \leq 0.05)$ & 0.12 & 0.11 & 0.82 & 1.34 & 34.55 & 12.26 \\
\hline $\mathrm{N}_{0}$ & $15.38 \pm 0.25 \mathrm{~d}$ & $2.17 \pm 0.09 \mathrm{~d}$ & $14.76 \pm 0.47 \mathrm{~b}$ & $25.21 \pm 1.37 \mathrm{~d}$ & $321 \pm 7.10 \mathrm{c}$ & $76.05 \pm 2.32 \mathrm{~d}$ \\
$\mathrm{~N}_{1}$ & $17.54 \pm 0.20 \mathrm{c}$ & $2.43 \pm 0.08 \mathrm{c}$ & $15.12 \pm 0.87 \mathrm{~b}$ & $31.81 \pm 2.12 \mathrm{c}$ & $424 \pm 8.27 \mathrm{~b}$ & $106.21 \pm 3.08 \mathrm{c}$ \\
$\mathrm{N}_{2}$ & $19.07 \pm 0.24 \mathrm{~b}$ & $2.66 \pm 0.02 \mathrm{~b}$ & $15.85 \pm 1.33 \mathrm{a}$ & $34.56 \pm 1.30 \mathrm{~b}$ & $487 \pm 9.89 \mathrm{a}$ & $134.80 \pm 4.60 \mathrm{~b}$ \\
$\mathrm{~N}_{3}$ & $20.25 \pm 0.26 \mathrm{a}$ & $2.92 \pm 0.01 \mathrm{a}$ & $15.98 \pm 1.56 \mathrm{a}$ & $36.12 \pm 2.49 \mathrm{a}$ & $514 \pm 9.94 \mathrm{a}$ & $148.92 \pm 5.21 \mathrm{a}$ \\
\hline $\mathrm{LSD}(\mathrm{p} \leq 0.05)$ & 0.16 & 0.09 & 0.69 & 1.18 & 28.22 & 9.38 \\
\hline Irrigations & $*$ & $*$ & $*$ & $*$ & $*$ & $*$ \\
Nitrogen & $*$ & $*$ & $*$ & & $*$ & $*$ \\
Irrigations & $\mathrm{NS}$ & $\mathrm{NS}$ & $\mathrm{NS}$ & $\mathrm{NS}$ & $\mathrm{NS}$ & $\mathrm{NS}$ \\
nitrogen & & & & & $*$ \\
\hline
\end{tabular}

Values (means \pm S.E.) sharing a letter in common within a column do not differ significantly ( $\leq \leq 0.05)$. ${ }^{*}$ Significant; ${ }^{\text {NS}}$ Non-Significant. Pooled data for two years have been presented because year effect was non-significant. $\mathrm{I}_{1}=$ three leaf stage (V1), nine leaf stage (V2), tasseling (T) and milking stage $(\mathrm{M}) ; \mathrm{I}_{2}=\mathrm{V} 1, \mathrm{~V} 2, \mathrm{~T}, \mathrm{M}$ and dough stage $(\mathrm{R} 1), \mathrm{I}_{3}=\mathrm{V} 1, \mathrm{~V} 2, \mathrm{~T}, \mathrm{M}, \mathrm{R} 1$ and blister stage $(\mathrm{R} 2)$ with $70 \mathrm{~mm}$ water application depth. $\mathrm{N}_{0}=0$ $\mathrm{kg} \mathrm{N} \mathrm{ha}{ }^{-1} ; \mathrm{N}_{2}=150 \mathrm{~kg} \mathrm{~N} \mathrm{ha}^{-1} ; \mathrm{N}_{3}=200 \mathrm{~kg} \mathrm{~N} \mathrm{ha}^{-1}$ and $\mathrm{N}_{4}=250 \mathrm{~kg} \mathrm{~N} \mathrm{ha}^{-1}$.

Maize photosynthetic and transpiration rate at vegetative and reproductive stages are presented in figure 2 (a-c). At both stages, maximum transpiration rates and photosynthetic activities were recorded at $\mathrm{I}_{3}$ while minimum was recorded at $\mathrm{I}_{1}$. Furthermore, nitrogen application rates also significantly affected transpiration and photosynthetic rates. $\mathrm{N}_{3}$ level of nitrogen resulted in highest transpiration and photosynthetic activity while lowest was recorded at $\mathrm{N}_{0}$ (control). Overall, photosynthetic and transpiration activity was higher at vegetative stage compared with reproductive stage. 
a)

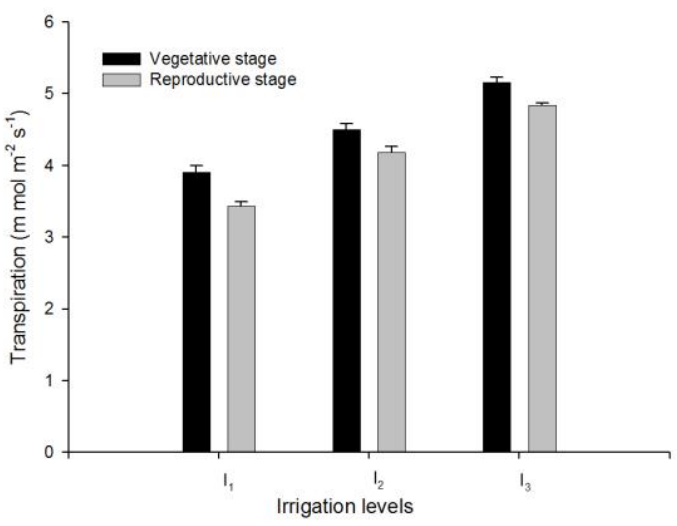

c)

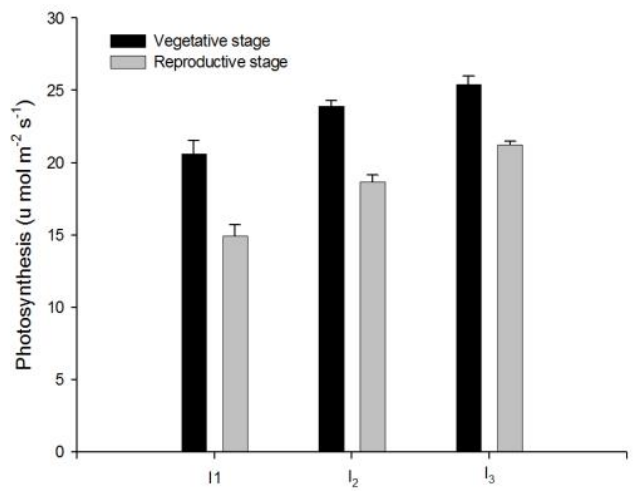

b)

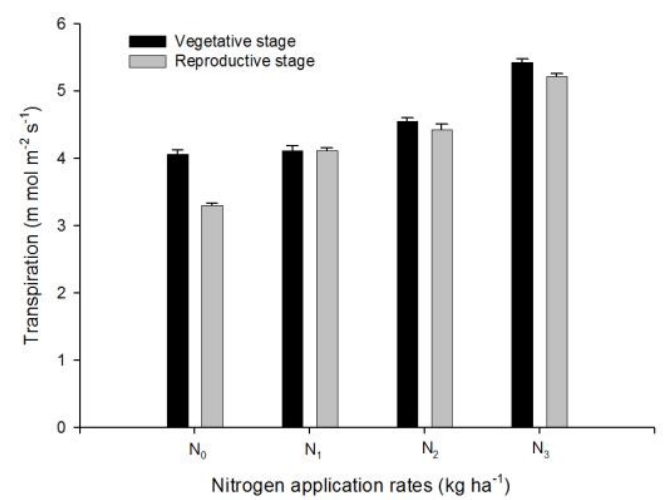

d)

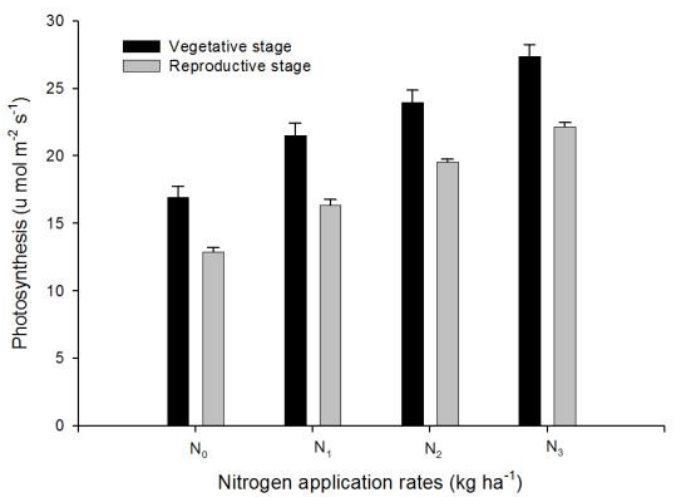

Figure 2. Influence of irrigation and nitrogen the (a-b) transpiration and (c-d) photosynthesis of maize at both vegetative and reproductive stages. Pooled data for two years have been presented because year effect was non-significant. Capped bars are S.E. of three replicates. $\mathrm{I}_{1}=$ three leaf stage $(\mathrm{V} 1)$, nine leaf stage $(\mathrm{V} 2)$, tasseling $(\mathrm{T})$ and milking stage $(\mathrm{M}) ; \mathrm{I}_{2}=\mathrm{V} 1, \mathrm{~V} 2, \mathrm{~T}, \mathrm{M}$ and dough stage (R1), I $=$ V1, V2, T, M , R1 and blister stage (R2) with $70 \mathrm{~mm}$ water application depth. $\mathrm{N}_{0}=0 \mathrm{~kg} \mathrm{~N} \mathrm{ha}^{-1} ; \mathrm{N}_{2}=150 \mathrm{~kg}$ $\mathrm{N} \mathrm{ha}^{-1} ; \mathrm{N}_{3}=200 \mathrm{~kg} \mathrm{~N} \mathrm{ha}^{-1}$ and $\mathrm{N}_{4}=250 \mathrm{~kg} \mathrm{~N} \mathrm{ha}^{-1}$.

The interaction between irrigation and nitrogen application rates also affected some other growth (plant height, LAI and CGR), yield and yield related characters (100-grain weight, grain and biological yield as well as harvest index) of maize considerably. With increasing irrigations and nitrogen application dosage, growth and yield of maize significantly improved. Plant height, LAI, CGR, 100-grain weight, grain yield, biological yield and harvest index was increased up to $30 \%, 54 \%, 46 \%, 33 \%$, $13 \%$, while almost double grain and biological yield was reaped at $\mathrm{I}_{3}-\mathrm{N}_{3}$ compared with $\mathrm{I}_{1}-\mathrm{N}_{0}$, respectively (Table $3)$. Moreover, $\mathrm{I}_{3}$ level of irrigation proved effective even at lower nitrogen rates $\left(\mathrm{N}_{1}, \mathrm{~N}_{2}\right)$ than higher nitrogen dose $\left(N_{3}\right)$ under $I_{1}$, however, in case of $I_{2}$ some of the growth, yield and related attributes were statistically similar with $\mathrm{I}_{3}$ even at $\mathrm{N}_{2}$. For example, at $\mathrm{I}_{2}-\mathrm{N}_{2}$, and $\mathrm{I}_{2}-\mathrm{N}_{3}$ the values of grain yield ( $8.6 \pm 0.62$ and $8.10 \pm 0.49$, respectively) and biological yield $(17.9 \pm 1.27,18.4 \pm 0.95)$ were statistically at par with $\mathrm{I}_{3}-\mathrm{N}_{1}$ and $\mathrm{I}_{3}-\mathrm{N}_{2}(7.8 \pm 0.54$ and $8.9 \pm 0.28)$ for grain yield and $\mathrm{I}_{3}-\mathrm{N}_{2}$ and $\mathrm{I}_{3}-\mathrm{N} 3(17.9 \pm 0.57$ and
$19.9 \pm 0.60$ ), for biological yield respectively. The interaction of $I_{1}$ with all levels of nitrogen produced lower plant produce than other levels of irrigation with their respective nitrogen application rates (Table 3 ).

WUE and NUE increased linearly as irrigations were increased from $I_{1}$ to $I_{3}$. Maximum irrigations $\left(I_{3}\right)$ resulted in higher water and nitrogen use efficiencies than $I_{1}$ and $I_{2}$, respectively at maximum nitrogen level, confirming them as yield limiting factors under semi-arid conditions. However, regarding $\mathrm{N}$ application rates, $\mathrm{N}_{3}$ level of nitrogen showed maximum NUE at $\mathrm{I}_{3}$ than other $\mathrm{N}$ rates $\left(\mathrm{N}_{1}\right.$ and $\left.\mathrm{N}_{2}\right)$. Further, at $\mathrm{I}_{2}-\mathrm{N}_{2}$ the NUE was statistically similar to $\mathrm{I}_{3}-\mathrm{N}_{2}$ while higher than $\mathrm{I}_{2}-\mathrm{N}_{3}$ (Table 3; Fig. 3d).

Significant and positive linear relationships were observed between 100-grain weight vs grain yield $\left(\mathrm{R}^{2}>\right.$ $91 \%)$ and biological yield vs grain yield $\left(\mathrm{R}^{2}>99 \%\right)$ at all irrigation levels as well as biological yield vs harvest index $\left(\mathrm{R}^{2}>58 \%\right)$ at $\mathrm{I}_{1}$ and $\left(\mathrm{R}^{2}>93\right)$ at other two $\left(\mathrm{I}_{2}\right.$ and $\left.\mathrm{I}_{3}\right)$ irrigation levels (Fig. 3a-c). 
Table 3. Plant height, LAI, CGR, 100-grain weight, grain and biological yield harvest index of maize as well as water use efficiency as affected by different irrigation and nitrogen levels

\begin{tabular}{|c|c|c|c|c|c|c|c|c|c|}
\hline Treatments & & $\begin{array}{c}\text { Plant height } \\
\text { (cm) }\end{array}$ & $\begin{array}{l}\text { Max. Leaf } \\
\text { area index }\end{array}$ & $\begin{array}{c}\text { Max. Crop } \\
\text { Growth Rate } \\
\left(\mathbf{g ~ m}^{-2} \mathbf{d}^{-1}\right)\end{array}$ & $\begin{array}{l}\text { 100-grain } \\
\text { weight } \\
\text { (g) }\end{array}$ & $\begin{array}{l}\text { Grain yield } \\
\qquad\left(\mathbf{t} \mathbf{h a}^{-1}\right)\end{array}$ & $\begin{array}{c}\text { Biological } \\
\text { yield }\left(\mathrm{t} \mathrm{ha}^{-1}\right)\end{array}$ & $\begin{array}{c}\text { Harvest } \\
\text { index } \\
(\%)\end{array}$ & $\begin{array}{c}\text { Water use } \\
\text { efficiency } \\
\left(\mathrm{kg} \mathrm{ha}^{-1} \mathbf{~ m m}^{-}\right. \\
1)\end{array}$ \\
\hline \multirow[t]{4}{*}{$\mathrm{I}_{1}$} & $\mathrm{~N}_{0}$ & $157.0 \pm 0.57 \mathrm{j}$ & $3.52 \pm 0.011$ & $11.92 \pm 0.12 \mathrm{i}$ & $22.17 \pm 0.11 \mathrm{j}$ & $4.6 \pm 0.07 \mathrm{~g}$ & $9.9 \pm 0.11 \mathrm{f}$ & $43.67 \pm 0.32 \mathrm{f}$ & $10.29 \pm 0.75 \mathrm{~g}$ \\
\hline & $\mathrm{N}_{1}$ & $169.0 \pm 0.99 \mathrm{~g}$ & $4.16 \pm 0.02 \mathrm{i}$ & $14.96 \pm 0.08 \mathrm{f}$ & $23.45 \pm 0.13 \mathrm{~h}$ & $5.7 \pm 0.10 \mathrm{f}$ & $12.0 \pm 0.23 \mathrm{e}$ & $45.43 \pm 0.09 \mathrm{e}$ & $11.05 \pm 0.60 \mathrm{fg}$ \\
\hline & $\mathrm{N}_{2}$ & $174.7 \pm 0.67 \mathrm{f}$ & $4.45 \pm 0.04 \mathrm{~g}$ & $15.61 \pm 0.07 \mathrm{~d}$ & $26.15 \pm 0.07 \mathrm{e}$ & $7.0 \pm 0.09 \mathrm{de}$ & $14.9 \pm 0.23 \mathrm{~cd}$ & $45.56 \pm 0.17 \mathrm{e}$ & $11.29 \pm 0.37 \mathrm{f}$ \\
\hline & $\mathrm{N}_{3}$ & $179.7 \pm 0.89 \mathrm{e}$ & $4.88 \pm 0.09 \mathrm{~d}$ & $16.60 \pm 0.04 \mathrm{~b}$ & $27.16 \pm 0.10 \mathrm{~d}$ & $8.5 \pm 0.13 \mathrm{bc}$ & $18.0 \pm 0.34 \mathrm{ab}$ & $45.57 \pm 0.31 \mathrm{e}$ & $11.09 \pm 0.26 \mathrm{fg}$ \\
\hline \multirow[t]{4}{*}{$\mathrm{I}_{2}$} & $\mathrm{~N}_{0}$ & $160.3 \pm 0.85 \mathrm{i}$ & $3.78 \pm 0.01 \mathrm{k}$ & $12.20 \pm 0.01 \mathrm{~h}$ & $22.87 \pm 0.05 \mathrm{i}$ & $4.7 \pm 0.10 \mathrm{~g}$ & $9.9 \pm 0.23 \mathrm{f}$ & $45.34 \pm 0.11 \mathrm{e}$ & $11.03 \pm 0.27 \mathrm{fg}$ \\
\hline & $\mathrm{N}_{1}$ & $172.3 \pm 0.80 \mathrm{~g}$ & $4.52 \pm 0.07 \mathrm{~h}$ & $15.30 \pm 0.04 \mathrm{e}$ & $24.43 \pm 0.09 \mathrm{~g}$ & $6.3 \pm 0.29 \mathrm{ef}$ & $13.1 \pm 0.67 \mathrm{de}$ & $46.33 \pm 0.29 \mathrm{~d}$ & $11.02 \pm 0.42 \mathrm{fg}$ \\
\hline & $\mathrm{N}_{2}$ & $187.3 \pm 0.33 \mathrm{c}$ & $4.72 \pm 0.06 \mathrm{f}$ & $16.27 \pm 0.01 \mathrm{c}$ & $27.05 \pm 0.06 \mathrm{~d}$ & $8.6 \pm 0.62 \mathrm{bc}$ & $17.9 \pm 1.27 \mathrm{ab}$ & $46.81 \pm 0.20 \mathrm{~cd}$ & $11.81 \pm 0.28 \mathrm{ef}$ \\
\hline & $\mathrm{N}_{3}$ & $195.0 \pm 0.58 \mathrm{~b}$ & $5.22 \pm 0.01 \mathrm{~b}$ & $16.50 \pm 0.07 \mathrm{~b}$ & $28.41 \pm 0.10 \mathrm{~b}$ & $8.10 \pm 0.49 \mathrm{~b}$ & $18.4 \pm 0.95 \mathrm{ab}$ & $47.25 \pm 0.22 \mathrm{c}$ & $12.51 \pm 0.46 \mathrm{de}$ \\
\hline \multirow[t]{4}{*}{$\mathrm{I}_{3}$} & $\mathrm{~N}_{0}$ & $163.3 \pm 0.81 \mathrm{~h}$ & $3.99 \pm 0.08 \mathrm{j}$ & $12.61 \pm 0.03 \mathrm{~g}$ & $23.46 \pm 0.04 \mathrm{~h}$ & $4.2 \pm 0.14 \mathrm{~g}$ & $9.6 \pm 0.32 \mathrm{f}$ & $46.68 \pm 0.24 \mathrm{~cd}$ & $12.89 \pm 0.53 \mathrm{~cd}$ \\
\hline & $\mathrm{N}_{1}$ & $176.7 \pm 0.66 \mathrm{f}$ & $4.81 \pm 0.06 \mathrm{e}$ & $15.53 \pm 0.02 \mathrm{de}$ & $25.36 \pm 0.13 \mathrm{f}$ & $7.8 \pm 0.54 \mathrm{~cd}$ & $15.7 \pm 1.11 \mathrm{c}$ & $48.25 \pm 0.38 \mathrm{~b}$ & $13.67 \pm 0.38 \mathrm{bc}$ \\
\hline & $\mathrm{N}_{2}$ & $183.7 \pm 0.88 \mathrm{~d}$ & $5.12 \pm 0.03 \mathrm{c}$ & $16.40 \pm 0.08 \mathrm{bc}$ & $28.06 \pm 0.08 \mathrm{c}$ & $8.9 \pm 0.28 \mathrm{~b}$ & $17.9 \pm 0.57 \mathrm{ab}$ & $48.42 \pm 0.05 b$ & $13.92 \pm 0.48 \mathrm{~b}$ \\
\hline & $\mathrm{N}_{3}$ & $203.7 \pm 1.20 \mathrm{a}$ & $5.37 \pm 0.08 \mathrm{a}$ & $17.01 \pm 0.07 \mathrm{a}$ & $29.58 \pm 0.68 \mathrm{a}$ & $10.1 \pm 0.29 \mathrm{a}$ & $19.9 \pm 0.60 \mathrm{a}$ & $49.41 \pm 0.04 a$ & $15.46 \pm 0.64 \mathrm{a}$ \\
\hline $\mathrm{LSD}(\mathrm{p} \leq 0.05)$ & & 2.56 & 0.07 & 0.20 & 0.28 & 0.96 & 2.04 & 0.74 & 0.82 \\
\hline Irrigations & & $*$ & $*$ & $*$ & $*$ & $*$ & $*$ & $*$ & $*$ \\
\hline Nitrogen & & $*$ & $*$ & $*$ & $*$ & $*$ & $*$ & $*$ & $*$ \\
\hline Irrigations $\times \mathrm{n}$ & rogen & $*$ & $*$ & $*$ & $*$ & $*$ & $*$ & $*$ & $*$ \\
\hline
\end{tabular}

Values (means \pm S.E.) sharing a letter in common within a column do not differ significantly ( $\mathrm{p} \leq 0.05)$. ${ }^{\text {Significant; }}$ NSNon-Significant. Pooled data for two years have been presented because year effect was nonsignificant. $\mathrm{I}_{1}=$ three leaf stage $(\mathrm{V} 1)$, nine leaf stage $(\mathrm{V} 2)$, tasseling $(\mathrm{T})$ and milking stage $(\mathrm{M}) ; \mathrm{I}_{2}=\mathrm{V} 1, \mathrm{~V} 2, \mathrm{~T}, \mathrm{M}$ and dough stage $(\mathrm{R} 1)$, $\mathrm{I}_{3}=\mathrm{V} 1, \mathrm{~V} 2, \mathrm{~T}, \mathrm{M}, \mathrm{R} 1$ and blister stage $(\mathrm{R} 2)$ with $70 \mathrm{~mm}$ water application depth. $\mathrm{N}_{0}=0 \mathrm{~kg} \mathrm{Nha}^{-1} ; \mathrm{N}_{2}=150 \mathrm{~kg} \mathrm{Nha}^{-1} ; \mathrm{N}_{3}=200 \mathrm{~kg} \mathrm{Nha}^{-1}$ and $\mathrm{N}_{4}=250 \mathrm{~kg} \mathrm{~N} \mathrm{ha}^{-1}$. 
a)

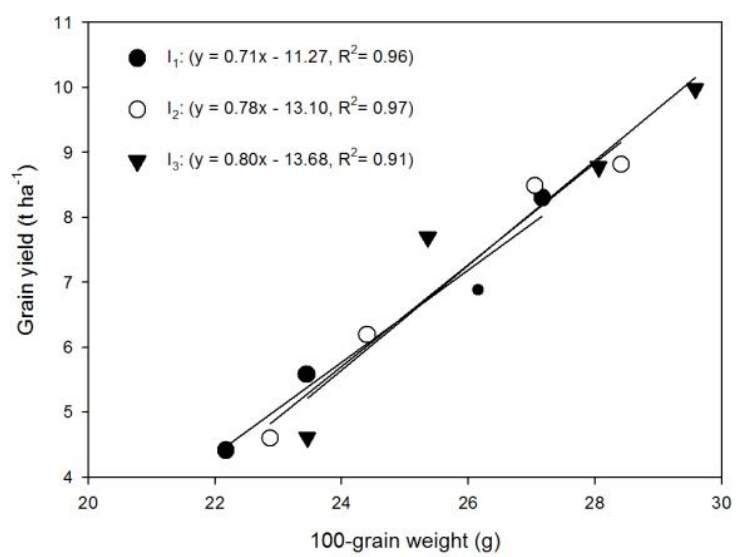

b)

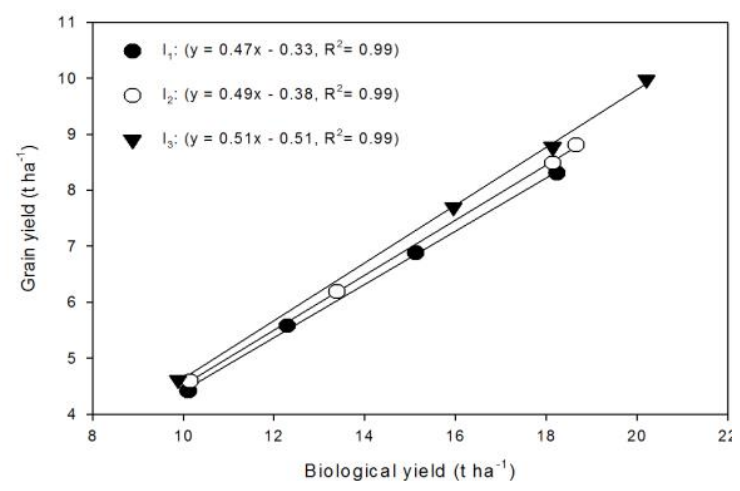

c)

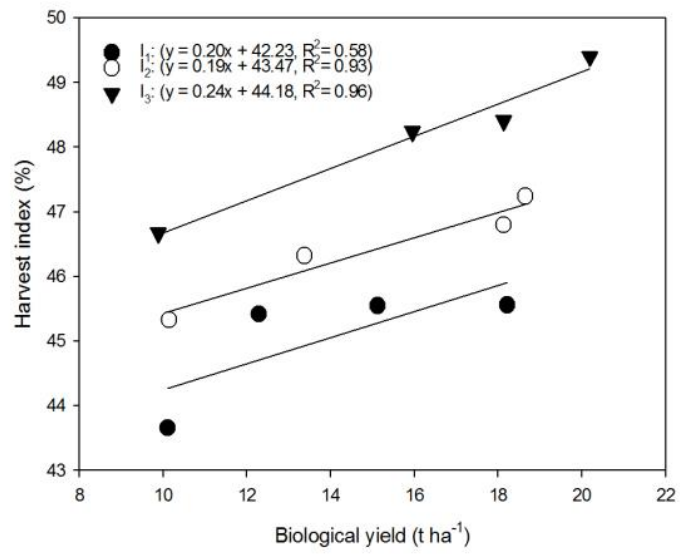

d)

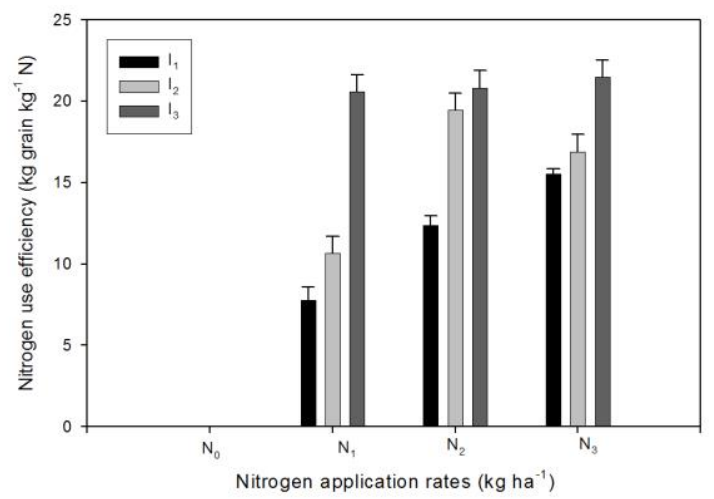

Figure 3. Relationships of (a) 100-grain weight vs grain yield, (b) biological yield vs grain yield, (c) biological yield vs harvest index using polynomial linear regression analysis, and (d) nitrogen use efficiency at different irrigation levels. Capped bars are S.E. of three replicates. $\mathrm{I}_{1}=$ three leaf stage $(\mathrm{V} 1)$, nine leaf stage $(\mathrm{V} 2)$, tasseling $(\mathrm{T})$ and milking stage $(\mathrm{M}) ; \mathrm{I}_{2}=\mathrm{V} 1, \mathrm{~V} 2, \mathrm{~T}, \mathrm{M}$ and dough stage (R1), $I_{3}=\mathrm{V} 1, \mathrm{~V} 2, \mathrm{~T}, \mathrm{M}, \mathrm{R} 1$ and blister stage (R2) with $70 \mathrm{~mm}$ water application depth. $\mathrm{N}_{0}=0 \mathrm{~kg} \mathrm{~N} \mathrm{ha}^{-1} ; \mathrm{N}_{2}=150 \mathrm{~kg} \mathrm{~N}^{-1}$; $\mathrm{N}_{3}=200 \mathrm{~kg} \mathrm{~N} \mathrm{ha}^{-1}$ and $\mathrm{N}_{4}=250 \mathrm{~kg} \mathrm{~N} \mathrm{ha}^{-1}$.

\section{DISCUSSION}

Irrigation at critical stages of a crop and availability of nutrients within the root zone furnishes better growth, development and ultimate yield. In this study, the effects of different irrigation schedules and nitrogen application rates were assessed on maize performance under field conditions. Higher nitrogen rates with maximum irrigations delayed the durations of phenological development in maize (Table 1). This could be explained by the fact that more availability of nitrogen to the plants and optimum soil moisture favored the crop vegetative growth yet resulted in delayed maturity. Suitable moisture conditions might promote root growth to procure more nutrients from the soil which results in improvement in plant development and vegetative growth and delays maturity. In contrast, treatments with low irrigation levels and reduced nitrogen doses resulted in early maturity. Low levels of irrigation might cause drought conditions thus forced the maize plants to mature earlier. These results are corroborated with the previous literature (Di Paolo and Rinaldi, 2008; Amin, 2011; Nsanzabaganwa et al., 2014). Amanullah et al. (2009) also stated that increased $\mathrm{N}$ levels delayed maturity in maize.
More number of irrigations and higher rates of nitrogen improved growth attributes of maize i.e., plant height, cob length, cob diameter, LAI, CGR (Table 2\&3). Water application and nitrogen affect crop growth through various mechanisms. $\mathrm{N}$ improves leaves chlorophyll contents and triggers cell division thus results in better plant growth (Qadri et al., 2015). Further, nitrogen application at higher rates might improve leaf morphology, leaf nitrogen contents, and developed basic infrastructural frame and led to enhanced photosynthetic efficiency thus amplified plant growth effectively. However, low water levels caused drought conditions during vegetative growth thus reduced leaf area and plant growth rate (Hammad et al., 2011; Zamir et al., 2014). Water deficit conditions at vegetative and reproductive stages reduced plant growth and development (Moser et al., 2006; Anjum et al., 2011). Furthermore, increased N rates improved maize growth, physiological indices, root development and yield formation of maize (Khan et al., 2012; Zhang et al., 2014).

In this experiment water levels and nitrogen fertilization greatly influenced the yield and yield related attributes of maize and nitrogen use efficiency as well. This confirms the progressive effect of soil water on 
nitrogen accessibility and plant's aptitude to uptake water and nitrogen simultaneously that led to their most effective usage when both are at an adequate level. An increase in plant growth with increased water and $\mathrm{N}$ levels might be due to better LAI and CGR values, which eventually yielded more grain and biological yield. Under water deficit conditions lower WUE limited N uptake and severely reduced grains rows/cob, grains/row, grains/cob, grain weight/cob (Table 2), 100-grain weight, grain yield, biological yield, harvest index (Table 3 ) and nitrogen use efficiency (Fig. 3d ). Crop might experience drought conditions at crop critical stages due to water limited environments that resulted in yield loss and reduced water and nitrogen use efficiency. Irrigation shortage at reproductive stages endured more yield reduction and reduced NUE than vegetative stages when results compared for adequate water vs. water deficit and adequate $\mathrm{N}$ vs. $\mathrm{N}$ deficit conditions at vegetative and reproductive stages under semi-arid climatic conditions (Mansouri-Far et al., 2010). Nutrient uptake and their use efficiencies are important regarding growth and yield of maize, however, limited water supply with lower rates of nitrogen results in reduced root growth with minimum nutrient uptake from soil profiles and minimum yield harvest, whereas under limited water conditions, severe yield loss in maize can be avoided with regular irrigations at flowering and grain-filling stages even though water supply is limited at vegetative stages (Igbadun et al., 2007). Furthermore, treatments with higher irrigations performed well due to ample supply of water necessary for the plant growth and yield formation. These results are in line with Gheysari et al. (2015) who found a significant reduction in 1000-grain weight and grain yield of maize when water stress was imposed during reproductive phase in maize. Stomatal closure, reduced photosynthesis and hampered respiratory activities due to water stress along with reduced $\mathrm{N}$ levels led to significant yield reduction in maize (Hammad et al., 2012). Moreover, reduced water and nitrogen availability cramped photosynthesis and transpiration rates at both vegetative and reproductive stages (Fig. 2). Water stress condition induced stomatal closure that led to reduced working efficiency of photosynthetic machinery and transpiration rates. However, lower photosynthesis and transpiration at reproductive stage than vegetative might be due to age factor and reduced leaf area after silking. Farooq et al. (2009) also argued that water stress significantly reduced transpiration and photosynthetic activities in maize. Additionally, reduced $\mathrm{N}$ dose also affected the photosynthetic and transpiration rates that led to reduced crop produce. So higher $\mathrm{N}$ rates might be used to combat this problem by keeping in mind that too higher rates of $\mathrm{N}$ may lead to reduced NUE that will not only costs much but may also cause yield penalty (Di Paolo and Rinaldi, 2008; Gheysari et al., 2009). Moreover, Kuscu et al. (2013) obtained enhanced grain yield with increased amounts of water in maize however, higher water use efficiency (WUE) and irrigation water use efficiency (IWUE) were recorded under $25 \%$ water deficit conditions than normal.
Furthermore, a non-linear, curvilinear and linear relationships between irrigation and maize yield was also reported by (Farre' and Faci 2006; Shahrokhnia and Sepaskhah, 2012). Usually, irrigation dynamics affect nitrogen use efficiency by regulating its uptake mechanisms. Nitrogen in $\mathrm{NH}_{4}{ }^{+}$and $\mathrm{NO}_{3}{ }^{-}$get dissolved in water (present in the soil solution) and then translocates from soil solution towards roots, shoots, leaves and other plant parts. Water thus helps $\mathrm{N}$ uptake by plants and had a direct relation to $\mathrm{N}$-uptake and overall NUE. Reduced NUE under less irrigation is the mirror image of what was found by Zand-Parsa et al. (2006) and Mahbod et al. (2015). They argued that irrigation affects nitrogen uptake by plants and its accumulation mechanisms in grains while the mechanism of $\mathrm{N}$ uptake by grains responds similar as plant $\mathrm{N}$ uptake to different irrigation levels.

In general, NUE response to irrigation treatments and $\mathrm{N}$ application rates seemed to be related to increased grain yield. However, increase $\mathrm{N}$ rates to enhance crop productivity is not an effective stratagem in agricultural ecosystems (Gheysari et al., 2009; Hammad et al., 2012) as it results in a significant reduction in NUE. Too high or too low $\mathrm{N}$ application cannot be accumulated efficiently thus a specific rate of $\mathrm{N}$ at suitable soil water levels may result an increase in NUE (Di Paolo and Rinaldi, 2008).

\section{CONCLUSIONS}

In crux, suitable nitrogen and water applications rates are the mainstream to get maximum yield and to harvest more nutrients from the soil. In this study, the best response of maize under semi-arid conditions was obtained with six irrigations and $250 \mathrm{~kg} \mathrm{ha}^{-1}$ nitrogen application in each year. Both WUE and NUE were increased with increase in irrigations while skipping irrigations at reproductive stages resulted in reduced growth and yield of maize. However, nitrogen and water application should be at their optimal levels because their application beyond the required limits not only results in yield reduction but also causes nutrient losses that have their own economic and environmental implications.

\section{LITERARURE CITED}

Abbas, F. and A. Fares. 2009. Best management practices to minimize nonpoint-source pollution in agriculture. College of Tropical Agriculture and Human Resources, University of Hawaii at Manoa, Manoa, Hawaii, USA. http://www.ctahr.hawaii.edu/oc/freepubs/pdf/SCM-26.pdf.

Amanullah, R.A., S.K. Khattak and R.A. Khattak. 2009. Effects of plant density and $\mathrm{N}$ on phenology and yield of maize. $\mathrm{J}$ Plant Nutr. 32(3):245-259.

Amin, M.E.H. 2011. Effect of different nitrogen sources on growth, yield and quality of fodder maize (Zea mays L.). J. Saudi Soc. Agric. Sci.10:17-23.

Anjum, S.A., L.C. Wang, M. Farooq, M. Hussain, L. L. Xue and C.M. Zou. 2011. Brassinolide application improves the drought tolerance in maize through modulation of enzymatic antioxidants and leaf gas exchange. J. Agron. Crop Sci. 197:177-185.

Aynehband, A., M. Valipoor and E. Fateh. 2011. Stem reserve accumulation and mobilization in wheat (Triticum aestivum L.) as affected by sowing date and N-P-K levels under Mediterranean conditions. Turk J Agri For. 35:319-331. 
Di Paolo, E. and M. Rinaldi. 2008. Yield response of corn to irrigation and nitrogen fertilization in a Mediterranean environment. Field Crops Res. 105:202-210.

Dobermann, A. 2007. Nutrient use efficiency-measurement and management. In: Proceedings of the IFA International Workshop on Fertilizer Best Management Practices, Brussels, Belgium.

Farooq, M., A. Wahid, N. Kobayashi, D. Fujita and S.M.A. Basra. 2009. Plant drought stress: effects, mechanisms and management. Agron. Sustain. Dev. 29:185-212.

Farre', I. and J.M. Faci. 2006. Comparative response of maize (Zea mays L.) and sorghum (Sorghum bicolor L. Moench) to deficit irrigation in a Mediterranean environment. Agric. Water Manage. 83:135-143.

Gheysari, M, H.W. Loescher, S. H. Sadeghi, S. M. Mirlatifi, M. J.Z. and G. Hoogenboom. 2015. Water-yield relations and water use efficiency of maize under nitrogen fertigation for semiarid environments: experiment and synthesis. Adv. Agron. 130:175-229.

Gheysari, M., S.M. Mirlatifi, M. Bannayan, M. Homaee and G. Hoogenboom. 2009. Interaction of water and nitrogen on maize grown for silage. Agric. Water Manage. 96:809-821.

Glamoclija, D., S. Jankovic, S. Rakic, R. Maletic, J. Ikanovic and Z. Lakic. 2011. Effects of nitrogen and harvesting time on chemical composition of biomass of Sudan grass, fodder sorghum, and their hybrid. Turk. J. Agri. For. 35:127-138.

Halvorson, A.D., A.R. Mosier, C.A. Reule and W.C. Bausch 2006. Nitrogen and tillage effects on irrigated continuous corn yields. Agron. J. 98:63-71.

Hammad, H.M., A. Ahmad, A. Wajid and J. Akhter. 2011. Maize response to time and rate of nitrogen application. Pak. J. Bot. 43:1935-1942.

Hammad, H.M., A. Ahmad, F. Abbas and W. Farhad. 2012. Optimizing water and nitrogen use for maize production under semiarid conditions. Turk. J. Agri. For. 36:519-532.

Homer, D.C. and Pratt, P.F. 1961. Methods of Analysis for Soils, Plants and Waters. Div. of Agriculture Science, University of California, Davis.

Hunt, R. 1978. Plant Growth Analysis. Edward Arnold, London, UK.

Igbadun, H.E., Tarimo, A.K.P.R., Salim, B.A. and Mahoo, H.F 2007. Evaluation of selected crop water production functions for an irrigated maize crop. Agric. Water Manage. 94: 1-10.

Islam, M.R., S.M.E. Rahman, M.M. Rahman, D.H. Oh and C.S. Ra. 2010. The effects of biogas slurry on the production and quality of maize fodder. Turk. J. Agri. For. 34:91-99.

Kuscu, H., A. Karasu, M. Oz, A.O. Demir and I. Turgut. 2013. Effect of irrigation amounts applied with drip irrigation on maize evapotranspiration, yield, water use efficiency, and net return in a sub-humid climate. Turk. J Field Crops 18:13-19.

Khaliq, T., A. Ahmad, A. Hussain and M.A. Ali. 2009. Maize hybrids response to nitrogen rates at multiple locations in semiarid environment. Pak. J. Bot. 41:207-224.

Khan M.B., F. Yousaf, M. Hussain, M.W. Haq, D. J. Lee and M. Farooq. 2012. Influence of planting methods on root development, crop productivity and water use efficiency in maize hybrids. Chilean J. Agric. Res. 72:556-563.
Li, H., L. Li, T. Wegenast, C.F. Longin, X. Xu, A.E. Melchinger and S. Chen. 2010. Effect of N supply on stalk quality in maize hybrids. Field Crop Res. 118:208-214.

Mahbod, M., S. Zand-Parsa and A. R. Sepaskhah. 2015. Modification of maize simulation model for predicting growth and yield of winter wheat under different applied water and nitrogen. Agric. Water Manage. 150:18-34.

Mansouri-Far, C., S.A.M.M. Sanavy and S.F. Saberali. 2010. Maize yield response to deficit irrigation during lowsensitive growth stages and nitrogen rate under semi-arid climatic conditions. Agric Water Manage. 97:12-22.

Mohamed, M.Y. 2010. Development and stability of some Sudanese sunflower hybrids under irrigated conditions. Helia 33:135-144.

Moser, S.B., B. Feil, S. Jampatong and P. Stamp. 2006. Effects of pre-anthesis drought, nitrogen fertilizer rate, and variety on grain yield, yield components, and harvest index of tropical maize. Agric. Water Manage. 81:41-58.

Nsanzabaganwa, E., T.K. Das, D.S. Rana and S.N. Kumar. 2014. Nitrogen and phosphorus effects on winter maize in an irrigated agroecosystem in western Indo-Gangetic plains of India. Maydica 59:152-160.

Payero, J.O., S.R. Melvin, S. Irmak and D. Tarkalson. 2006. Yield response of corn to deficit irrigation in a semiarid climate. Agric. Water Manage. 84:101-112.

Qadri, R.W.K., I. Khan, M.M. Jahangir, U. Ashraf, G. Samin, A. Anwer, M. Adnan and M. Bashir. 2015. Phosphorous and foliar applied nitrogen improved productivity and quality of potato. Am. J. Plant Sci. 6:144-149.

Sepaskhah, A.R., S. Fahandezh-Saadi and S. Zand-Parsa. 2011. Logistic model application for prediction of maize yield under water and nitrogen management. Agric. Water Manage. 99: 51-57.

Shahrokhnia, M.H. and A.R. Sepaskhah. 2012. Evaluation of wheat and maize evapotranspiration determination by direct use of Penman-Monteith in a semi-arid region. Arch Agron Soil Sci 58:1283-1302.

Watson, D.J. 1952. The physiological basis of variation in yield. Adv Agron 4:101-145.

Zamir, S.I., M. Asif, Ihtisham-ul-haq, U. Ashraf, S. Hussain and M.K. Khan. 2014. Maize phenology, yield and its quality is affected by organic mulches and various irrigation regimes. Int. J. Mod. Agri. 3:56-59.

Zand-Parsa, S., A.R. Sepaskhah and A. Ronaghi. 2006. Development and evaluation of integrated water and nitrogen model for maize. Agric Water Manage. 81:227256.

Zhang, X., G. Huang and Q. Zhao. 2014. Differences in maize physiological characteristics, nitrogen accumulation, and yield under different cropping patterns and nitrogen levels. Chilean J. Agric. Res. 74:326-332. 